\title{
UTILIZAÇÃO DE MODELOS ALOMÉTRICOS PARA ESTIMAR A ÁREA FOLIAR DE Talinum triangulare E Talinum paniculatum
}

Ricardo Ferreira Oliveira ${ }^{1}$, Adriano Jakelaitis ${ }^{1 *}$, Ellen Carla Francisca Alexandre ${ }^{1}$, Leandro Spíndola Pereira $^{1}$, Márcia Nara da Silva ${ }^{2}$, Daniel Emanuel Cabral de Oliveira ${ }^{3}$, Gustavo Dorneles de Sousa ${ }^{1}$, Gustavo Silva de Oliveira ${ }^{1}$

RESUMO - O estudo da área foliar das plantas permite inferir sobre os parâmetros de crescimento e de desenvolvimento vegetal, tornando-a um importante indicativo da produtividade agrícola. Objetivou-se, determinar uma equação que permita estimar a área foliar de Talinum triangulare e T. paniculatum por intermédio das medidas lineares das folhas. Amostrou-se aleatoriamente duzentos limbos foliares de cada espécie e com auxílio de escalímetro digital determinou as dimensões foliares de comprimento (C), largura (L) e o seu produto (CL), de modo não destrutivo. Utilizando as mesmas folhas, obteve-se área foliar real (AFR) por meio do software ImageJ ${ }^{\circledR}$. Foram testados modelos lineares, geométricos e exponenciais e a equação selecionada foi a que apresentou menor valor de soma dos quadrados dos resíduos e maiores coeficientes de determinação $\left(\mathrm{R}^{2}\right)$. Os resultados obtidos neste estudo demonstram que para as duas espécies, equações de regressão lineares simples, utilizando o produto do comprimento pela máxima largura, dada por $S f_{\text {Ttriangulare }}=0,3247+0,6204 * \mathrm{CL}\left(\mathrm{R}^{2}=0,9662 *\right)$ e $S f_{\text {Tpaniculatum }}=$ $0,1357+0,6019 * \mathrm{CL}\left(\mathrm{R}^{2}=0,9948 *\right)$ são indicadas para expressar a estimativa da área foliar de T. triangulare e $T$. paniculatum, respectivamente.

Palavras chave: hortaliças não convencionais, medidas foliares, métodos não destrutivos.

\section{ALLOMETRIC MODELS TO ESTIMATE LEAF AREA IN Talinum triangulare AND Talinum paniculatum}

ABSTRACT - The measuring of leaf area allows inferring about plant growth and development, thus becoming
an important indicator of agricultural productivity. This study aimed to determine an equation for estimation
of leaf area in Talinum triangulare and T. paniculatum, as a function of leaf linear measurements. First, two
hundred leaf limbi of each species were randomly sampled and, with the aid of a digital scaler, leaf dimensions
such as length $(L)$, width $(W)$, and their product $(L W)$ were measured in a non-destructive manner. Then,
the real leaf area $(R L A)$, of the same leaves, was measured using Image $J^{\circledR}$ software. Linear, geometric,
and exponential models were tested; then, the selected equation was that showing the smallest sum of squared
residuals (SSR) and the highest determination coefficients $\left(R^{2}\right)$. For both species, simple linear regression
equations using the product between leaflength and maximum width were effective to estimate leaf area,
which were $S f T_{\text {triangulare }}=0.3247+0.6204 * L W\left(R^{2}=0.9662^{*}\right)$ and $S f T_{\text {paniculatum }}=0.1357+0.6019 * L W\left(R^{2}=0.9948^{*}\right)$ for T. triangulare and T. paniculatum, respectively.

Keywords: by-product, Lactuca sativa L., substrate.

\footnotetext{
${ }^{1}$ Instituto Federal de Educação, Ciência e Tecnologia Goiano (IF Goiano), Campus Rio Verde, Rio Verde, GO, Brasil. ricardooliveiraagroufg@hotmail.com, ellencarlabiologa@gmail.com, leandrop629@gmail.com,gustavodorneles7888@gmail.com, gustavosilvadeoliveira147@gmail.com.

${ }^{2}$ Universidade Federal de Goiás, UFG, Goiânia, GO, Brasil. marcianara@gmail.com.

${ }^{3}$ Instituto Federal de Educação, Ciência e Tecnologia Goiano (IF Goiano), Campus Iporá, Iporá, GO, Brasil. oliveira.d.e.c@gmail.com

*Contato para correspondência: adriano.jakelaitis@ifgoiano.edu.br.
} 


\section{INTRODUÇÃO}

Talinum triangulare (Jacq.) Willd. e Talinum paniculatum (Jacq.) Gaertn. pertencem a família Talinaceae (anteriormente Portulacaceae) (Brilhaus et al., 2016) e apresentam potencial agronômico e econômico, visto que suas folhas são vendidas como verduras não convencionais, principalmente em feiras situadas nas regiões do Norte, Nordeste e Centro-Oeste, por pequenos produtores, contribuindo para uma fonte alternativa de renda (Alexandre et al., 2018). No Brasil, T. triangulare é comumente conhecida por cariru, caruru e joão-gomes (Cardoso 1997; Cardoso et al., 2017; Coelho Netto et al., 2018; Madeira \& Botrel, 2018), enquanto T. paniculatum por major-gomes, língua-de-vaca, beldroega grande e maria-gorda (Vieira, 2014; Madeira \& Botrel, 2018).

Embora essas espécies sejam consideradas infestantes no território brasileiro (Huziwara et al., 2009; Borchartt et al., 2013; Carvalho, 2013), T. triangulare e T. paniculatum apresentam elevado potencial para o consumo humano, em virtude da sua qualidade nutricional (Kinupp \& Barros, 2008; Carvalho, 2009). Ademais, podem também serem utilizadas como plantas medicinais, como por exemplo, para acelerar a cicatrização de feridas (Thanamool et al., 2013; Reis et al., 2015).

Para um bom manejo vegetal, são necessários o conhecimento do seu comportamento de crescimento. Dessa forma, são necessários estudos básicos para determinar os parâmetros de crescimento, e isto requer informações que podem ser obtidas sem a necessidade de equipamentos sofisticados (Morgado et al., 2013). Dentre os diversos parâmetros, a área foliar é de fundamental importância (Duarte et al., 2009; Schmildt et al., 2017), já que a folha é o principal órgão envolvido no processo transpiratório, sendo responsável pelas trocas gasosas entre a planta e o ambiente, fatores estes, determinantes da produção (Morgado et al., 2013).

A estimativa da área foliar pode ser realizada por meio de instrumentos de medição (métodos destrutivos) ou por modelos de regressão (métodos não destrutivos) (Francisco et al., 2014). Os métodos de natureza destrutiva exigem a retirada da folha ou de outras estruturas, apresentando como desvantagem a não aplicação em estudos onde a quantidade de amostras é limitada. Diferentemente, nos métodos não destrutivos, as medidas são realizadas nas plantas, sem amostragens destrutivas, preservando sua integridade e permitindo a continuidade das medidas durante todo ciclo de desenvolvimento (Adami et al., 2008; Richter et al., 2014).

De forma não destrutiva, a área foliar pode ser obtida através de modelos de estimação em função das dimensões lineares das folhas e o seu produto (Toebe et al., 2012). O procedimento usual desse método envolve medidas de comprimento, largura e das áreas de folhas amostradas e, em seguida, obtenção dos coeficientes de determinação que relacionam as medidas lineares com a área da folha. Na literatura são descritas diversas equações matemáticas que são utilizadas com rapidez, precisão, confiabilidade e facilidade, possibilitando estudos de outra natureza na mesma planta (Sachet et al., 2015).

O estudo de área foliar pelo método não destrutivo apresenta vasta utilização, nas diversas espécies, como no feijão-de-porco (Toebe et al., 2012), feijão-caupi (Lima et al., 2017), soja (Adami et al., 2008), acerola (Lucena et al., 2011), girassol (Aquino et al., 2011), em estudos de plantas daninhas aquática e terrestre (Bianco et al., 2008; Marchi et al., 2011; Carvalho et al., 2011), plantas ornamentais (Silva et al., 2008) e medicinais (Candido et al., 2013; Silva et al., 2017).

Diante do exposto, o objetivo foi determinar uma equação que permita estimar a área foliar de $T$. triangulare e T. paniculatum por intermédio das medidas de dimensões de comprimento e largura das folhas.

\section{MATERIALE MÉTODOS}

O experimento foi realizado no setor de Olericultura do Instituto Federal Goiano em Rio Verde, Goiás, sob as coordenadas geográficas de latitude $17^{\circ} 48^{\prime} 55^{\prime \prime S ~ e ~}$ de longitude 50 $56^{\prime} 28^{\prime \prime} \mathrm{O}$, com altitude de $753 \mathrm{~m}$. Segundo a classificação de Koppen e Geiger, o clima local é tropical (Aw). As precipitações variam de 1.500 a 1.800 $\mathrm{mm}$ anuais e ocorre nos meses de outubro a maio, e os meses de seca são de junho a setembro. A variação da temperatura média anual é de 20 a $35^{\circ} \mathrm{C}$.

Sementes das espécies de $T$. triangulare e de $T$. paniculatum foram colocadas para germinar em bandejas de isopor de 288 células, utilizando o substrato comercial Bioplant ${ }^{\circledR}$ e cobertura com vermiculita. Após a germinação, quando as plântulas apresentavam seis folhas expandidas, aos 30 dias após a semeadura, as mudas foram transplantadas para os canteiros. Os canteiros foram feitos em campo aberto, sendo um para cada espécie. Cada canteiro apresentou dimensões 
de 1x24 metros. Os canteiros foram feitos com enxada rotativa e, por ocasião desta operação, foi feita a aplicação da adubação na dose de $600 \mathrm{~kg} \mathrm{ha}^{-1}$ da formulação 0414-08. O espaçamento adotado foi de $0,25 \times 0,25 m$ entre plantas. Aos 20 dias após transplantio foi aplicada a adubação de cobertura, utilizando $30 \mathrm{~kg}_{\text {de }} \mathrm{N} \mathrm{ha}^{-1}$, aplicado na forma de ureia. Os canteiros foram mantidos sem a presença de plantas daninhas por meio de capinas manuais.

Em dezembro de 2016, no início da fase reprodutiva, foram coletados aleatoriamente duzentos limbos foliares, totalmente expandidos e isentos de deformações, de ambas as espécies (T. triangulare e T. paniculatum), e logo após determinaram-se com auxílio de escalímetro digital em centímetros, as dimensões foliares (comprimento e largura), de modo não destrutivo. O comprimento (C) foi dimensionado ao longo da nervura principal entre a base e o de inserção do pecíolo até o seu ápice (Figura 1). Já para a largura (L), utilizou como referência

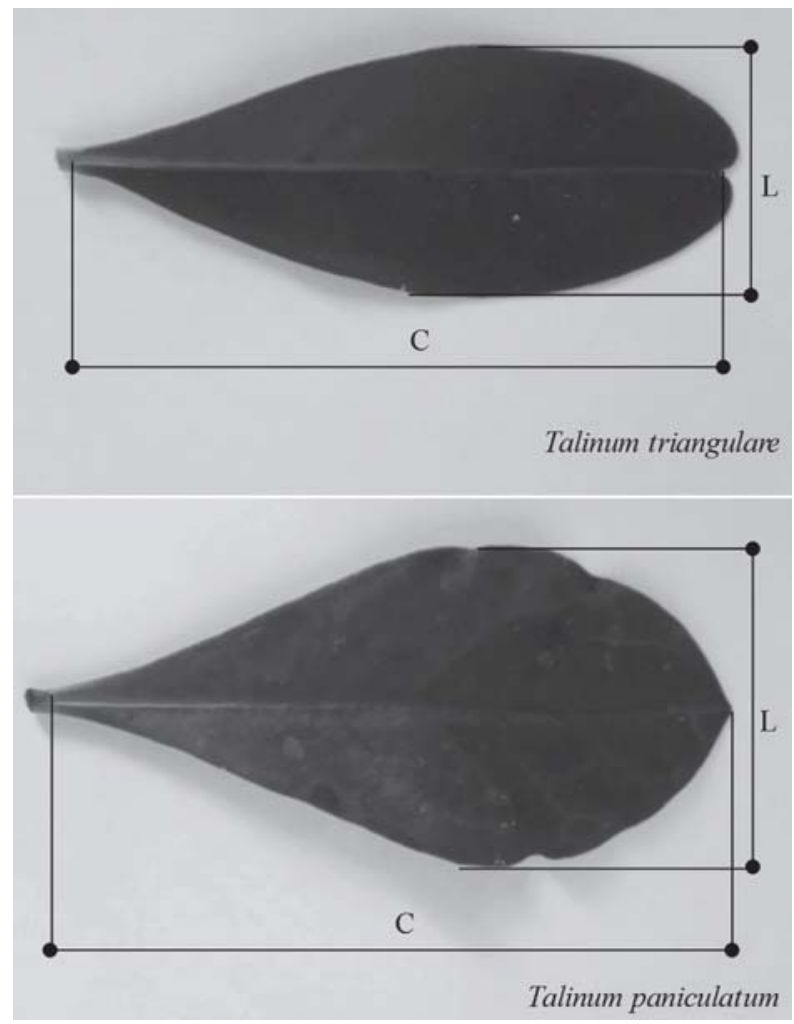

Figura 1 - Partes medidas nas folhas de Talinum triangulare e Talinum paniculatum para determinar o comprimento da nervura principal (C) e a maior largura da folha (L). a parte mediana da folha perpendicular à nervura principal (Bianco et al., 2002). Em seguida, utilizou as mesmas folhas, para obtenção da área foliar real (AFR) por meio do software Image ${ }^{\circledR}$ (Método destrutivo) (Martin et al., 2013).

Para modelar a AFR (variável dependente $=\mathrm{Y}$ ) em função do C, da L ou do C x L como variáveis independentes $(\mathrm{X})$, foram realizadas análises de regressão, utilizando equação linear $\mathrm{Y}=\mathrm{a}+\mathrm{bx}$; geométrica $\mathrm{Y}=\mathrm{ax} \mathrm{x}^{\mathrm{b}}$ e exponencial $\mathrm{Y}=\mathrm{ab}^{\mathrm{x}}$. No caso de $\mathrm{X}$ apresentarse idêntico ao produto, estabeleceu-se a equação linear passando pela origem, supondo que a área é proporcional a um retângulo ( $\mathrm{C}$ x L ). Primeiramente os dados foram analisados por meio de estatística descritiva, utilizando seus valores máximo, mínimo e médio por meio do aplicativo Excel ${ }^{\circledR}$. Logo após, com o aplicativo estatístico Sigmaplot ${ }^{\circledR}$, obteve-se as equações de regressão que estima AFR.

\section{RESULTADOS E DISCUSSÃO}

O resumo das medidas lineares obtidas nas folhas de $T$. triangulare e $T$. paniculatum de acordo com as duas metodologias utilizadas encontram-se na Tabela 1. Os valores de comprimento (C) das folhas de T. triangulare pelo método não destrutivo, variaram de 3,30 a 9,40 cm, com comprimento médio de $6,23 \mathrm{~cm}$, enquanto que a largura (L) do limbo foliar variou de 1,0 a 3,99 cm com largura média de 2,24 cm. Em relação a área foliar, estimada pelo modelo proposto, os resultados variaram de 2,62 a 22,85, com $\hat{Y}_{\mathrm{i}}$ média de 9,40 $\mathrm{cm}^{2}$ (Tabela 1).

Para os valores máximos, mínimos e médios do comprimento, largura e da área foliar de T. paniculatum, verificou, valor de C médio igual a 7,61 cm, variando de 3,58 a 12,56 cm, enquanto que para a largura, a variação foi de 2,05 a 6,17 cm e valor médio de 3,66 cm. Aárea foliar de $T$. paniculatum, estimada pelo modelo, apresentou variação de 4,57 a 46,78 cm² e área foliar média de $\hat{Y}_{i}=18,03 \mathrm{~cm}^{2}$ (Tabela 1 ). As caracteristicas de C, L e $\hat{Y}_{i}$ em estudos com Ageratum conyzoides (Bianco et al., 2008) foram próximas ao do presente trabalho. Para Francisco et al. (2014) a variabilidade das medidas lineares existentes respalda o indicativo que melhor será o desempenho do modelo gerado, pois permitirá sua utilização nos diferentes estágios de crescimento das plantas.

A distribuição percentual dos limbos foliares em relação às faixas permite descrever as características 
de tamanho das folhas. Na Figura 2 verifica-se para as duas metodologias aplicadas a distribuição percentual dos duzentos limbos foliares das espécies de $T$. triangulare (2A) e T. paniculatum (2B), em relação às faixas de tamanho.

Para T. triangulare, observa-se que, independentemente do método utilizado, cerca de $75 \%$ da área foliar está relacionada com folhas variando de 5 a $15 \mathrm{~cm}^{2}, 13 \%$ relaciona-se com folhas de 0 a $5 \mathrm{~cm}^{2}$, e os $12 \%$ restante, pelas folhas que apresentam faixas superiores a $15,1 \mathrm{~cm}^{2}$ (Figura 2A). Diferente de T. triangulare, T. paniculatum apresentou folhas de tamanho maiores e mais homogêneas, com a área foliar na faixa de 10,1 a $20 \mathrm{~cm}^{2}$, com 49,5\% da área foliar (Figura 2B).

Na Tabela 2 permite-se dizer que a utilização das dimensões lineares de comprimento, largura e o produto destas proporcionaram uma estimativa satisfatória da área foliar, para as diferentes espécies de Talinum. Para T. triangulare verifica-se que a maioria das equações estimadas apresentam elevados coeficientes de determinação, acima de 0,85 , o que permitem estimar a área foliar satisfatoriamente, exceto a equação $L$ linear $\left(\mathrm{R}^{2}=0,6111\right), \mathrm{L}$ geométrica $\left(\mathrm{R}^{2}=0,5961\right)$ e $\mathrm{L}$ exponencial $\left(\mathrm{R}^{2}=0,4065\right)$ que não explicam com precisão a área foliar real dessa espécie. Diferentemente, $T$. paniculatum apresentou-se com elevados coeficientes de determinação para todas as equações estimadas $\left(R^{2}>0,93\right)$, possibilitando uma melhor estimativa da área foliar.

A soma dos quadrados dos resíduos indica a dispersão das médias observadas em relação ao valor estimado (Bianco et al., 2002). A equação selecionada para estimativa da área foliar foi a que apresentou menor valor de soma dos quadrados dos resíduos. Já para a avaliação do desempenho dos modelos na comparação das estimativas, baseou-se no melhor coeficiente de determinação $\left(\mathrm{R}^{2}\right)$ da análise de regressão, entre os valores estimados de área foliar $\left(\hat{\mathrm{Y}}_{\mathrm{i}}\right)$, e os valores observados de área foliar real (AFR) da planta por meio do software Image $J^{\circledR}\left(Y_{i}\right)$. Desta forma, para as espécies de Talinum estudadas as melhores equações descritas são aquelas que envolvem o produto do comprimento pela largura máxima do limbo foliar, uma vez que, as mesmas obtiveram os maiores coeficientes de determinação aliado aos menores soma de quadrados do resíduo (Tabela 2).

Em estudos com as espécies de Ageratum conyzoides (Bianco et al., 2008), Passiflora spp. (Morgado et al., 2013), Combretum leprosum (Candido et al., 2013), Coffea arabica (Schmildt et al., 2014), Glycine max (Richter et al., 2014), Litchi chinensis (Oliveira et al., 2017), Theobroma cacao (Schmildt et al., 2017) os autores verificam que a utilização de equações que envolvem o produto do comprimento pela largura se torna a melhor opção para avaliar a área foliar, o que se deve, possivelmente, a um maior número de informações trazidas das dimensões foliares, considerando inclusive o efeito da variação métrica de ambas variáveis, uma vez que a folha é um órgão altamente plástico, apresentando variações nas suas proporções em resposta ao ambiente em que se encontra (Boeger et al., 2009).

Tabela 1 - Valores máximos, mínimos, e médios do comprimento (C), largura (L) e da área foliar dos limbos (Y e Ŷ) de Talinum triangulare e Talinum paniculatum pelo método destrutivo (MD) e não destrutivo (MND)

\begin{tabular}{|c|c|c|c|c|c|c|}
\hline \multirow{2}{*}{ Estatística } & \multicolumn{2}{|c|}{$\mathrm{C}(\mathrm{cm})$} & \multicolumn{2}{|c|}{$\mathrm{L}(\mathrm{cm})$} & \multicolumn{2}{|c|}{ Área foliar $\left(\mathrm{cm}^{2}\right)$} \\
\hline & $\mathrm{MD}^{1}$ & $\mathrm{MND}^{2}$ & MD & MND & $\mathrm{Y}_{\mathrm{MD}}$ & $\hat{\mathrm{Y}}_{\mathrm{MND}}$ \\
\hline \multicolumn{7}{|c|}{ Talinum triangulare } \\
\hline Médio & 6,21 & 6,23 & 2,30 & 2,24 & 9,59 & $9,40^{*}$ \\
\hline Máximo & 10,89 & 9,40 & 5,84 & 3,99 & 25,43 & $22,85^{*}$ \\
\hline Mínimo & 1,91 & 3,30 & 1,03 & 1,00 & 2,11 & $2,62 *$ \\
\hline \multicolumn{7}{|c|}{ Talinum paniculatum } \\
\hline Médio & 7,61 & 7,61 & 3,66 & 3,66 & 18,03 & $18,03^{*}$ \\
\hline Máximo & 12,56 & 12,56 & 6,27 & 6,17 & 48,88 & $46,78^{*}$ \\
\hline Mínimo & 3,58 & 3,58 & 1,79 & 2,05 & 4,56 & $4,57^{*}$ \\
\hline
\end{tabular}

1-Realizado pelo medidor eletrônico Image ${ }^{\circledR}$; 2-Realizado por meio do escalímetro digital. * Valor de área foliar estimado pelo modelo. 

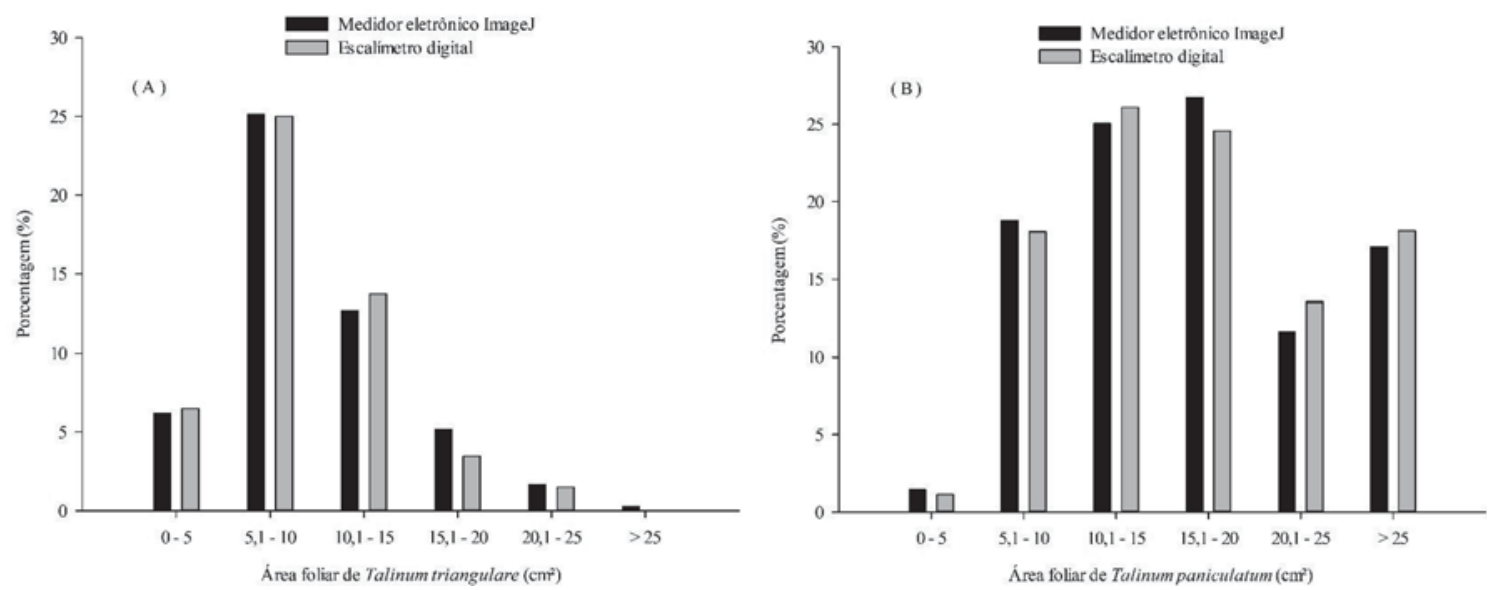

Figura 2 - Distribuição percentual dos limbos foliares de Talinum triangulare (A) e Talinum paniculatum (B) em relação às faixas de tamanho para duas metodologias aplicadas.

Tabela 2 - Equações de regressão estimadas da área foliar em função das medidas lineares do limbo foliar de Talinum triangulare e Talinum paniculatum

\begin{tabular}{|c|c|c|c|c|c|}
\hline $\mathrm{X}^{1}$ & Modelo & $\mathrm{R}^{2}$ & GL & SQR & Equação estimada (Sf) \\
\hline \multicolumn{6}{|c|}{ Talinun triangulare } \\
\hline $\mathrm{C}$ & Linear & $0,8520 *$ & 198 & 663,5416 & $-8,6613+2,9412 * \mathrm{C}$ \\
\hline $\mathrm{L}$ & Linear & $0,6111^{*}$ & 198 & 1743,9114 & $-1,9239+4,9963 * \mathrm{~L}$ \\
\hline CL & Linear & $0,9662 *$ & 198 & 151,4268 & $0,3247+0,6204 *(C L)$ \\
\hline $\mathrm{CL}(0,0)$ & Linear & $0,9653^{*}$ & 199 & 155,6756 & $0,6378 *(\mathrm{CL})$ \\
\hline $\mathrm{C}$ & Geométrica & $0,8934 *$ & 198 & 477,9392 & $0,2470 * C^{1,9737}$ \\
\hline $\mathrm{L}$ & Geométrica & $0,5961 *$ & 198 & 1810,7847 & $4,1240 * \mathrm{~L}^{1,0280}$ \\
\hline $\mathrm{C}$ & Exponencial & $0,8750 *$ & 198 & 560,5484 & $1,6961 * 1,3059^{\mathrm{C}}$ \\
\hline $\mathrm{L}$ & Exponencial & $0,4065^{*}$ & 198 & 2661,2979 & $5,0174 * 1,3201^{\mathrm{L}}$ \\
\hline \multicolumn{6}{|c|}{ Talinun paniculatum } \\
\hline $\mathrm{C}$ & Linear & $0,9440 *$ & 198 & 1030,7042 & $-18,6926+4,8227 * C$ \\
\hline $\mathrm{L}$ & Linear & $0,9593 *$ & 198 & 750,4052 & $-16,5777+9,4490 * \mathrm{~L}$ \\
\hline CL & Linear & $0,9948 *$ & 198 & 95,1788 & $0,1357+0,6019 *(C L)$ \\
\hline $\mathrm{CL}(0,0)$ & Linear & $0,9948 *$ & 199 & 95,9975 & $0,6055 *(\mathrm{CL})$ \\
\hline $\mathrm{C}$ & Geométrica & $0,9659 *$ & 198 & 627,8672 & $-5,8580 * C^{1,5500}$ \\
\hline $\mathrm{L}$ & Geométrica & $0,9763^{*}$ & 198 & 436,9847 & $1,8678 * L^{2,0819}$ \\
\hline $\mathrm{C}$ & Exponencial & $0,9322 *$ & 198 & 1248,0074 & $5,5426 * 1,3582^{\mathrm{C}}$ \\
\hline $\mathrm{L}$ & Exponencial & $0,9305^{*}$ & 198 & 1279,2204 & $6,2913 * 1,8490^{\mathrm{L}}$ \\
\hline
\end{tabular}

1 medidas lineares: comprimento (C) e largura (L) do limbo foliar; $\mathrm{R}^{2}$ : coeficientes de determinação; GL: graus de liberdade; SQR: somas de quadrados de desvios da regressão em escala original.

Na Figura 3 observa-se a relação linear entre a área foliar real (AFR) e o produto do comprimento (C) pela largura (L) do limbo foliar de T. triangulare e de T. paniculatum. Para Bianco et al. (2008) as equações geradas em trabalho com estes objetivos são imprescindíveis para análises de estudo de crescimento das espécies.
Na Figura 3A é possível verificar que a estimativa da área foliar de T. triangulare pode ser obtida pela equação linear $S f_{\text {Ttriangulare }}=0,3247+0,6204 * \mathrm{CL}$ com coeficiente de determinação de $\mathrm{R}^{2}=0,9662 *$. Deve-se ressaltar que as dispersões dos dados foram mínimas em relação à reta obtida, indicativo que a equação é recomendável para descrever de forma satisfatória a área foliar real. 

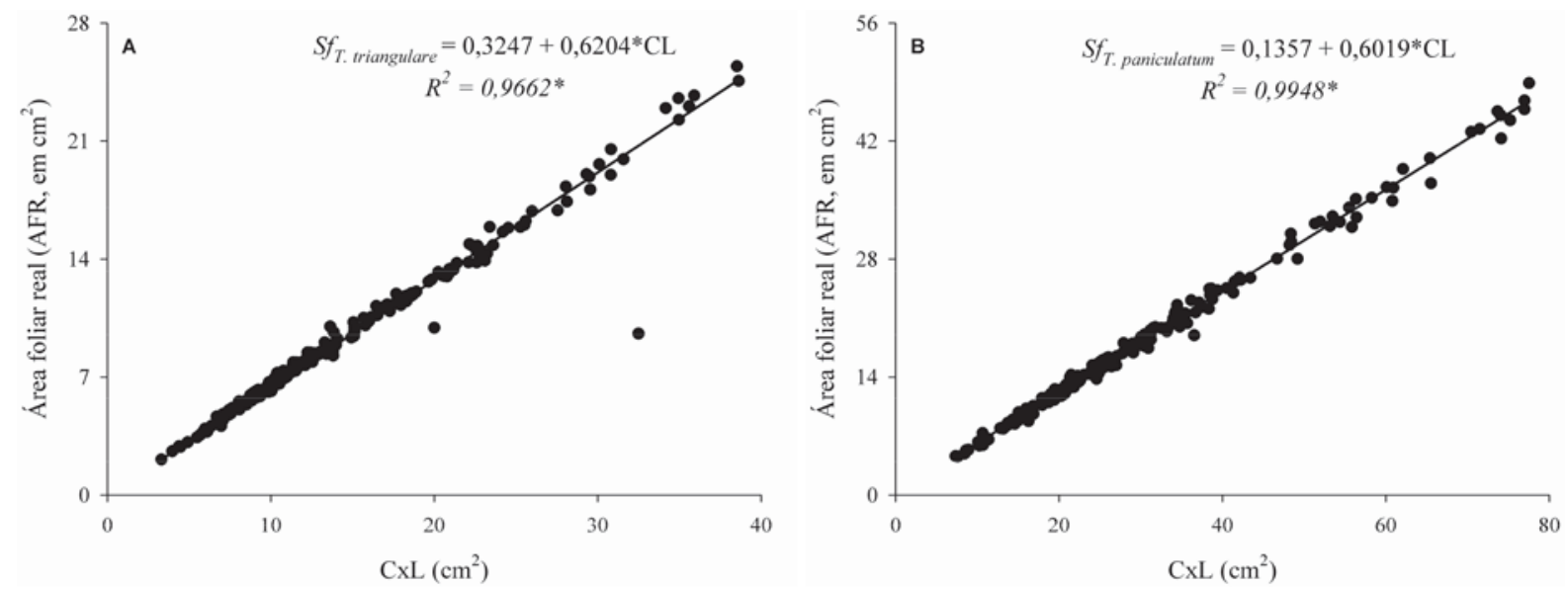

Figura 3 - Relação linear entre a área foliar real (AFR) e o produto do comprimento (C) pela largura (L) do limbo foliar de Talinun triangulare (A) e Talinun paniculatum (B).

Tais resultados recomendam que 96,62\% das variações analisadas podem ser explicadas pela equação estimada.

A equação estimada para T. paniculatum (Figura 3B) apresenta $\mathrm{R}^{2}=0,9948 *$, demonstrando que $99,48 \%$ das variações analisadas podem ser explicadas pela a seguinte equação: $S f_{\text {Tpaniculatum }}=0,1357+0,6019 * C L$. A reta obtida indica baixíssimas dispersões dos dados, o que permite descrever de forma confiável a área foliar real.

\section{CONCLUSÕES}

Para estimar a área foliar satisfatoriamente de $T$. triangulare e T. paniculatum podem ser utilizadas as equações lineares que envolvem o produto do comprimento pela largura.

As equações lineares $S f_{\text {Ttriangulare }}=0,3247+0,6204 * \mathrm{CL}$ e $S f_{\text {Tpaniculatum }}=0,1357+0,6019 *$ CL são indicadas para descrever de maneira confiável a área foliar de $T$. triangulare e T. paniculatum, respectivamente.

\section{AGRADECIMENTOS}

O presente trabalho foi realizado com apoio da Coordenação de Aperfeiçoamento de Pessoal de Nível Superior - Brasil (CAPES) - Código de Financiamento 001.

\section{LITERATURACITADA}

ADAMI, M.; HASTENREITER, F.A.; FLUMIGNAN, D.L. et al. Estimativa de área de folíolos de soja usando imagens digitais e dimensões foliares. Bragantia, v.67, n.4, p.1053-1058, 2008.
ALEXANDRE, E.F.; PEREIRA, L.S.; ANDRADE, J.W.S. et al. Plant biometric characterization and leaf micromorphometry of Talinum triangulare (Jacq.) Willd cultivated under shade. Revista Ceres, v.65, n.1, p.44-55, 2018.

AQUINO, L.A.; SANTOS JÚNIOR, V.C.; GUERRA, J.V.S. et al. Estimativa da área foliar do girassol por método não destrutivo. Bragantia, v.70, n.4, p.832-836, 2011.

BIANCO, S.; BIANCO, M.S.; CARVALHO, L.B. Estimativa da área foliar de Ageratum conyzoides usando dimensões lineares do limbo foliar. Acta Scientiarum. Agronomy, v.30, n.4, p.519523, 2008.

BIANCO, S.; PITELLI, R.A.; CARVALHO, L.B. et al. Estimativa da área foliar de Cissampelos glaberrima usando dimensões lineares do limbo foliar. Planta Daninha, v.20, n.3, p.353 -356, 2002.

BOEGER, M.R.T.; BIUZ, C.; GOLDENBERG, R. et al. Arquitetura foliar comparativa de Miconia sellowiana (DC.) Naudin (Melastomataceae) em diferentes fitofisionomias no Estado do Paraná, Brasil. Acta Botânica Brasílica, v.23, n.3, p.657-665, 2009.

BORCHARTT, L.; JAKELAITIS, A.; ASSIS VALADÃO, F.C. et al. Períodos de interferência de plantas daninhas na cultura do feijoeiro-comum (Phaseolus vulgaris L.). Revista Ciência Agronômica, v.42, n.3, p.725-734, 2011. 
BRILHAUS, D.; BRAUTIGAM, A.; METTLERALTMANN, T. et al. Reversible burst of transcriptional changes during induction of Crassulacean Acid Metabolism in Talinum triangulare. Plant Physiology, v.170, n.1, p.102-122, 2016.

CANDIDO, W.S.; COELHO, M.F.B.; MAIA, S.S.S. et al. Modelo para estimar a área foliar de Combretum leprosum Mart. Acta

Agronômica, v.62, n.1, p.37-41. 2013.

\section{CARDOSO, M.O. Cariru (Talinum} triangulare (Jacq.) Willd.). In: CARDOSO, M.O. (Ed.). Hortaliças não-convencionais da Amazônia. Brasília, DF: EMBRAPA-SPI, 1997. p. 39-45.

CARDOSO, M.O.; COHEN, A.; BERNI, R.F.; KANO, C. Consórcio couve-de-folha (Brassica oleracea var. acephala) e cariru (Talinum triangulare) sob duas alternativas de fertilização em cultivo protegido.

Horticultura Argentina, v.36, n.91, p.1851-9342, 2017.

CARVALHO, L.B. Classificações. In: CARVALHO, L.B. (Ed.) Plantas daninhas. Lages: FAESB, 2013. p.35-48.

CARVALHO, L.B.; SOUZA, M.C.; BIANCO, M.S. et al. Estimativa da área foliar de plantas daninhas de ambiente aquático: Pistia stratiotes. Planta Daninha, v.29, n.1, p.6568, 2011.

CARVALHO, R.D.S. Caracterização química e avaliação de folhas de Talinum patens Wand como complemento alimentar. Tese (Doutorado em Química), Salvador, BA: UFBA, 2009, 110 p.

COELHO NETTO, R.A.; PONTES, T.M.; SILVA FILHO, D.F. Hortaliças não convencionais: como cultivar. Manaus: Editora INPA, 2018, 33p.

DUARTE, D.J.; BIANCO, S.; LEONARDO, B.C. et al. Estimativa da área foliar de Euphorbia heterophylla. Planta Daninha, v.27, n.3, p.527-531, 2009.
FRANCISCO, J.P.; DIOTTO, A.V.; FOLEGATTI, M.V. et al. Estimativa da área foliar do abacaxizeiro cv. Vitória por meio de relações alométricas. Revista Brasileira de Fruticultura, v.36, n.2, p.285-293, 2014.

HUZIWARA, E.; OGLIARI, J.; FREITAS, S.P. et al. Levantamento fitossociológico de plantas daninhas na cultura da mandioca no município de Campos dos Goytacazes, RJ. Revista Raízes Amidos Tropicais, v.5, n.1, p.468-472, 2009.

KINUPP, V.F.; BARROS, I.B.I. Teores de proteína e minerais de espécies nativas, potenciais hortaliças e frutas. Ciência e Tecnologia Alimentos, v.28, n.4, p.846-857, 2008.

LIMA, C.J.G.S.; OLIVEIRA, F.A.; MEDEIROS, J.F. et al. Modelos matemáticos para estimativa de área foliar de feijão caupi. Revista Caatinga, v.21, n.1, p.120-127, 2017

LUCENA, R.R.M.; BATISTA, T.M.V.; DOMBROSKI. J.L.D. et al. Medição de área foliar de aceroleira. Revista Caatinga, v.24, n.2, p.40-45, 2011.

MADEIRA, N.R.; BOTREL, N. Talinum paniculatum e T. triangulare major-gomes e cariru. In: CORADIN, L.; CAMILLO, J.; PAREYN, F.; GERMAIN, C. (Ed.). Espécies nativas da flora brasileira de valor econômico atual ou potencial: plantas para o futuro: região Nordeste. Brasília, DF: Ministério do Meio Ambiente, 2018. p. 326-334.

MARCHI, S.R.; MARTINS, D.; COSTA, N.V. et al. Método não destrutivo de estimativa da área foliar de plantas daninhas de ambiente aquático: tanner-grass e capim fino. Semina: Ciências Agrárias, v.32, n.Spe, p.1717-1723, 2011.

MARTIN, T.N.; MARCHESE, J.A.; SOUSA, A.K.F. et al. Uso do software ImageJ na estimativa de área foliar para a cultura do feijão.

Interciencia, v.38, n.12, p.843-848, 2013.

MORGADO, M.A.D.O.; BRUCKNER, C.H.; ROSADO, L.D.S. et al. Estimação da área foliar por método não destrutivo, utilizando medidas lineares das folhas de espécies de Passiflora. Revista Ceres, v.60, n.5, p. 662-667, 2013. 
OLIVEIRA, P.S.; SILVA, W.; COSTA, A.A.M. et al. Leaf area estimation in litchi by means of allometric relationships. Revista Brasileira Fruticultura, v.39, n.Spe., e-403, 2017.

REIS, L.F.C.; CERDEIRA, C.D.; PAULA, B.F. et al. Chemical characterization and evaluation of antibacterial, antifungal, antimycobacterial, and cytotoxic activities of Talinum paniculatum. Revista do Instituto de Medicina Tropical de São Paulo, v.57, n.5, p.397-405. 2015.

RICHTER, G.L.; JÚNIOR, A.Z.; STRECK, N.A. et al. Estimativa da área de folhas de cultivares antigas e modernas de soja por método não destrutivo.

Bragantia, v.73, n.4, p.416-425, 2014.

SACHET, M.R.; PENSO, G.A.; PERTILLE, R.H. et al. Estimativa da área foliar de pessegueiro por método não-destrutivo. Ciência Rural, v.45, n.12, p.2161-2163, 2015.

SCHMILDT, E. R.; TREVISAN, E.; BELIQUE, M. et al. Modelos alométricos para determinação da área foliar de cacaueiro 'PH-16' em sombreamento e pleno sol. Revista Agroambiente, v.11, n.1, p.47-55, 2017.

SCHMILDT, E.R.; AMARAL, J.A.T.; SCHMILDT, O. et al. Análise comparativa de equações para estimativa da área foliar em cafeeiros. Coffee Science, v.9, n.2, p.155-167, 2014.
SILVA, S.F.; PEREIRA, L.R.; CABANEZ, P.A. et al. Modelos alométricos para estimativa da área foliar de boldo pelo método não destrutivo. Revista Agrarian, v.10, n.37, p.193-198, 2017.

SILVA, S.H.M.G.; LIMA, J.D.; BENDINI, H.N. et al. Estimativa da área foliar do antúrio com o uso de funções de regressão. Ciência Rural, v.38, n.1, p.243-246, 2008.

THANAMOOL, C.; PAPIROM, P.; CHANLUN, S. et al. Talinum paniculatum (Jacq.) Gaertn: a medicinal plant with potential estrogenic activity in ovariectomized rats. International Journal of Pharmacy and Pharmaceutical Sciences, v.5, n.2, p.478485. 2013.

TOEBE, M.; FILHO, A.C.; BURIN, C. et al. Modelos para estimação da área foliar de feijãode-porco por dimensões foliares. Bragantia, v.71, n.1, p.37-41. 2012.

VIEIRA, A.C. Atividade antibacteriana e características químicas e fitoquímicas de Talinum paniculatum (JACQ) GAERTN. (major-gomes). Dissertação (Mestrado em Ciência e Tecnologia de Alimentos), Porto Alegre, RS: UFRGS, 2014, $53 \mathrm{p}$.

Recebido para publicação em 14/12/2018 e aprovado em 20/3/2019. 